Fixed Point Theory, 20(2019), No. 1, 31-58

DOI: $10.24193 /$ fpt-ro.2019.1.03

http://www.math.ubbcluj.ro/ nodeacj/sfptcj.html

\title{
CARISTI-LIKE CONDITION. EXISTENCE OF SOLUTIONS TO EQUATIONS AND MINIMA OF FUNCTIONS IN METRIC SPACES
}

\author{
A.V. ARUTYUNOV*, B.D. GEL'MAN**, E.S. ZHUKOVSKIY*** AND S.E. ZHUKOVSKIY**** \\ *Peoples' Friendship University of Russia, 6 Miklukho-Maklaya st, Moscow, Russia, 117198 \\ Lomonosov Moscow State University, \\ 1 Leninskie Gory, Moscow, Russia, 119992 \\ Institute for Information Transmission Problems of RAS (Kharkevich Institute), \\ 19 Bol'shoi Karetnyi per., Moscow, Russia, 127051. \\ V.A. Trapeznikov Institute of Control Sciences of RAS, \\ 65 Profsoyuznaya st., Moscow, Russia, 117997. \\ E-mail: arutun@orc.ru \\ **Voronezh State University, 1 Universitetskaya pl, Voronezh, Russia, 394006 \\ E-mail: gelman_boris@mail.ru \\ ***Tambov State University, 33 International'naya st, Tambov, Russia, 392622 \\ E-mail: zukovskys@mail.ru \\ ****V.A. Trapeznikov Institute of Control Sciences of RAS, \\ 65 Profsoyuznaya st., Moscow, Russia, 117997. \\ E-mail: s-e-zhuk@yandex.ru
}

\begin{abstract}
In the paper, there were studied Caristi-like conditions that guaranteed existence of a minimum of a function on a metric space. For functions dependent on a parameter, there were obtained conditions for existence of a minimum for each value of the parameter. These results were applied to derive conditions for fixed point and coincidence point existence for mappings in metric spaces. For mappings dependent on a parameter, there were obtained conditions of coincidence point existence for each value of the parameter.

Key Words and Phrases: Caristi-like conditions, coincidence point, fixed point.

2010 Mathematics Subject Classification: 54H25, 49J53, 47H10.

Acknowledgements. The results of Sections 1,3 and 4 are due to the first and the second authors, who were supported by a grant from the Russian Science Foundation (project no. 17-11-01168). The investigation was supported by the RFBR grants (projects no. 17-51-12064, 18-01-00106, 17-01-00553) and by the Volkswagen Foundation.

\section{REFERENCES}

[1] A.V. Arutyunov, Caristi's condition and existence of a minimum of a lower bounded function in a metric space. Applications to the theory of coincidence points, Proc. of the Steklov Inst. of Math., 291(2015), no. 1, 24-37.
\end{abstract}


[2] A.V. Arutyunov, On the existence of solutions of nonlinear equations, Doklady Mathematics, 95(2017), no. 2, 1-4.

[3] A.V. Arutyunov, B.D. Gel'man, Minimum of a functional in a metric space and fixed points, Comp. Math. \& Math. Physics, 49(2009), no. 7, 1111-1118.

[4] J.-P. Aubin, I. Ekeland, Applied Nonlinear Analysis, John Wiley \& Sons, 1984.

[5] J. Caristi, Fixed point theorems for mappings satisfying inwardness conditions, Trans. Amer. Math. Soc., 215(1976), 241-251.

[6] F.H. Clarke, Optimization and Nonsmooth Analysis, John Wiley \& Sons, 1983.

[7] A. Granas, Continuation method for contractive maps, Topol. Methods Nonlinear Anal., 3(1994), no. 2, 375-379.

[8] A. Granas, J. Dugundji, Fixed Point Theory, Springer-Verlag, 2003.

[9] R. Precup, On the continuation method and the nonlinear alternative for Caristi-type non-selfmappings, J. Fixed Point Theory Appl., 16(2014), no. 1, 3-10.

[10] W. Takahashi, Minimization theorems and fixed point theorems, Nonlinear Anal. \& Math. Economics, 829(1993), 175-191.

[11] A.V. Arutyunov, R.B. Vinter, A simple finite approximations proof of the Pontryagin maximum principle under reduced differentiability hypotheses, Set-Valued Analysis, 12(2004), 5-24.

[12] E.R. Avakov, A.V. Arutyunov, E.S. Zhukovskii, Covering mappings and their applications to differential equations unsolved for the derivative, Differential Equations, 45(2009), no. 5, 627649.

[13] A.V. Arutyunov, E.S. Zhukovskiy, S.E. Zhukovskiy, Coincidence points principle for mappings in partially ordered spaces, Topology and its Appl., 179(2015), no. 1, 13-33.

[14] A.V. Arutyunov, S.E. Zhukovskiy, Existence of local solutions in constrained dynamic systems, Applicable Anal., 90(2011), no. 6, 889-898.

Received: March 27, 2017; Accepted: July 5, 2017. 
